\title{
MECANISMO DE TRANSMISIÓN DE CARGAS PERPENDICULARES AL PLANO DEL MURO EN MUROS DE MAMPOSTERÍA NO REFORZADA
}

\author{
Juan Diego Jaramillo ${ }^{(1)}$
}

\begin{abstract}
RESUMEN
Este informe es parte de un proyecto de investigación que pretende estudiar los mecanismos de resistencia sísmica de la mampostería no reforzada. En lo que sigue se explora un mecanismo de transmisión de cargas inerciales perpendiculares al plano de muros de mampostería no reforzada que se presenta como promisorio para tratar de explicar el comportamiento adecuado o no de algunas de estas edificaciones cuando son sometidas a sismos intensos. Los resultados del modelo propuesto se comparan con los resultados del ensayo en mesa vibradora de dos muros a escala natural instrumentados con sensores extensométricos, acelerómetros y medidores de desplazamiento. Los resultados analíticos coinciden bastante bien con los resultados experimentales.
\end{abstract}

\section{SUMMARY}

This report is part of a research project intended to study the seismic resistance mechanisms of unreinforced masonry. A new mechanism for transmitting inertial loads acting perpendicular to the plane of masonry walls is explored. The proposed mechanism is promissory for explaining the adequacy of some of these types of construction when subjected to intense earthquakes. Analytical results of the proposed mechanism are compared to experimental results obtained from walls tested in a shaking table and instrumented with strain gauges, accelerometers and displacement sensors. Two full scale walls were tested in the shaking table and the experimental results agreed very well with the analytical predictions.

\section{INTRODUCCIÓN}

Actualmente, la mampostería no reforzada está prohibida como sistema estructural por una gran cantidad de códigos de construcción sismo resistente en zonas de amenaza sísmica intermedia y alta a lo largo del mundo; entre ellos se incluye el de Colombia desde su versión inicial en 1984.

Las experiencias de sismos intensos recientes en Colombia muestran una vulnerabilidad media de este tipo de construcciones, pero no es muy claro si este nivel de vulnerabilidad se debe a los materiales en si, a una mala disposición de los elementos dentro de la estructura, a malos detalles de conexión con otros elementos importantes dentro de la estructura como el sistema de techo, o a una combinación de los anteriores.

Artículo recibido el 9 de noviembre de 2001 y aprobado para su publicación el 5 de septiembre de 2002. Se aceptarán comentarios y/o discusiones hasta cinco meses después de su publicación.

${ }^{(1)}$ Departamento de Ingeniería Civil, Universidad EAFIT, A.A. 3300, Medellín, Colombia, E-mail: jjarami@eafit.edu.co 
Es claro, también, de los escenarios de daños por sismos recientes que, en un mismo sector dentro de una ciudad fuertemente golpeada por un sismo, algunas edificaciones de mampostería no reforzada sufren daños severos e incluso colapso, mientras que algunas otras no presentan daño alguno. Este hecho apoya la idea de que con determinadas disposiciones de elementos dentro de la estructura, cuidando los detalles de conexión entre los elementos e incorporando un sistema de control de calidad durante la ejecución de la obra es posible alcanzar niveles de vulnerabilidad aceptables para edificaciones de mampostería no reforzada de baja altura. Este objetivo es particularmente atractivo en un país como Colombia donde gran cantidad de la población vive en situaciones económicas precarias y donde no siempre se logra implementar el tipo de construcción de mampostería no reforzada pero confinada tal como lo estipula el código.

Este informe es parte de un proyecto de investigación que pretende estudiar los mecanismos de resistencia sísmica de la mampostería no reforzada. En lo que sigue se explora un mecanismo de transmisión de cargas inerciales perpendiculares al plano de muros de mampostería no reforzada que se presenta como promisorio para tratar de explicar el comportamiento adecuado o no de algunas de estas edificaciones cuando son sometidas a sismos intensos.

\section{PLANTEAMIENTO DEL MODELO}

En lo que sigue se hará referencia a la nomenclatura definida en las figs. 1 y 2 . En la fig. 1 se muestra la orientación del muro en estudio respecto a la aceleración solicitante y los ejes de referencia, $X, Y$ y $Z$ que indican: la dirección perpendicular al plano del muro, la dirección horizontal a lo largo del muro y la dirección vertical, respectivamente.

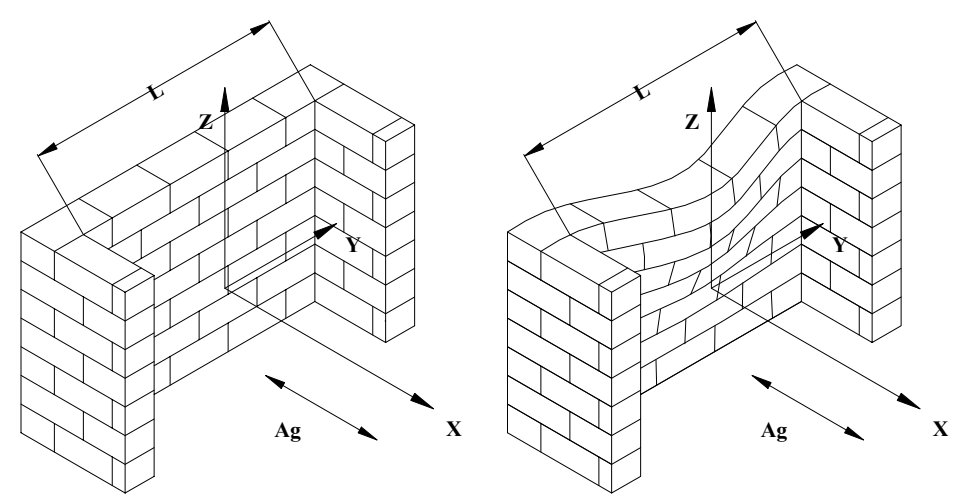

Figura 1. Orientación del muro respecto a la aceleración en la base y deformación de éste

Cuando el muro se somete a una aceleración en la base $A_{g}$, las fuerzas inerciales que se generan debido a la masa distribuida del muro hacen que este se deforme por fuera del plano como se ilustra en la fig. 1. Un corte vertical a través del centro del muro muestra que el muro se deforma en doble curvatura y ésta es casi nula en el tramo superior. Un corte horizontal cercano al borde libre, en la parte superior del muro, indica que el muro también se deforma en doble 
curvatura y la máxima aparece en el centro del muro. Lo anterior indica que en el tramo superior del muro, la mayoría de las fuerzas inerciales se transmite hacia los costados de este.

El mecanismo de transferencia de las cargas inerciales en la parte superior del muro se podría simplificar diciendo que todas las fuerzas se transmiten hacia los costados, y este mecanismo sólo puede existir si se resiste flexión en el eje $Z$ y corte en el plano $X Y$.

En la fig. 2 se muestra una sección del muro de mampostería en la que se indica la traba de los ladrillos y el mortero de pega horizontal y vertical, de espesores b y h, respectivamente. Igualmente, se señalan las dimensiones del ladrillo: longitud, $l$, ancho, $t, \mathrm{y}$ altura, $w, \mathrm{y}$ nuevamente los ejes de referencia, $X, Y$ y $Z$.

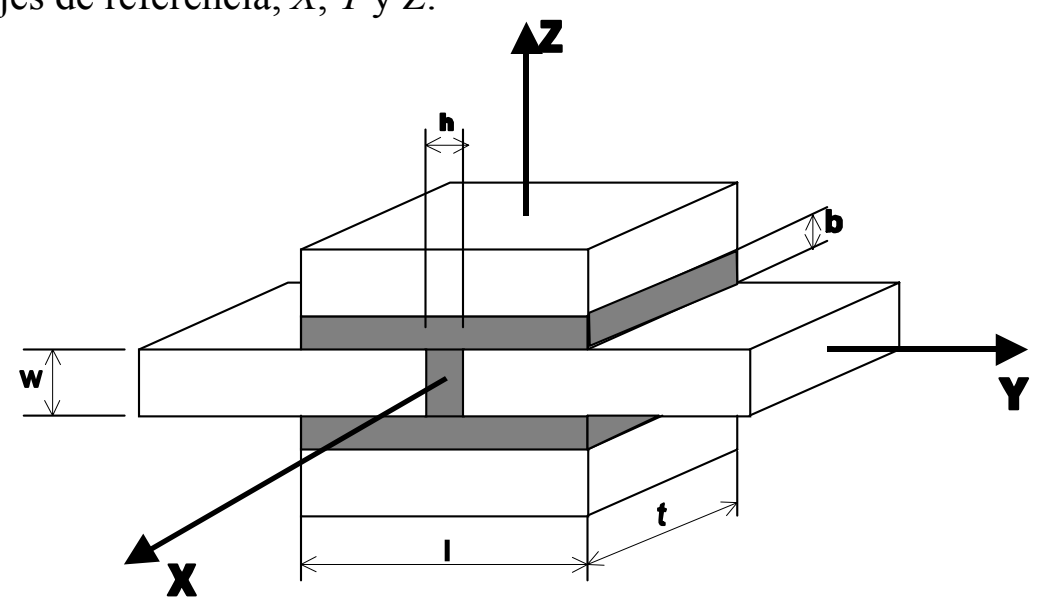

Figura 2. Sección de muro de mampostería.

Si se desprecia la resistencia a la tracción del mortero de pega en muros de mampostería no reforzada, hipótesis tradicionalmente aceptada $\mathrm{y}$, como se verá más adelante, sin mucha influencia en el modelo de transmisión de cargas que se plantea, y si además, se considera que el muro no tiene carga vertical ni restricción al desplazamiento vertical (muro en voladizo) que habilite la transmisión de momentos flexionantes en esta dirección por la existencia de una precompresión (Paulay y Priestley, 1992), o el efecto de arco en dirección vertical (Abrams et al., 1993) -este caso se presenta con frecuencia en los muros no cargueros del último nivel de una casa habitación tradicional-, y finalmente, si se considera que el muro está apoyado en muros perpendiculares según se muestra en la figura 1, que tampoco representan una restricción lateral importante que habilite el mecanismo de arco en dirección horizontal, se llega a la conclusión de que en caso de que esta disposición de muros resista alguna aceleración sísmica, como en realidad ocurre, se necesita la habilitación de un mecanismo de transmisión de carga diferente a los postulados hasta ahora que expliquen las resistencias reportadas (ver por ejemplo Paulay y Priestley, 1992; Abrams et al., 1993; Lam, Wilson, y Hutchinson, 1995; Shultz y Mueffelman, 1999; Gambarotta y Moneto, 1999; Kuzik, Cheng y Elwi, 1999; Mojsilovic y Marti, 1999; Blaikie y Davey, 2000; y Doherty et al., 2000). En todos estos artículos se postula el mecanismo de transmisión de cargas en dirección vertical para acciones actuando en dirección perpendicular al muro, y en algunos, el efecto combinado de arco en dos direcciones perpendiculares, habilitado 
por la restricción a las deformaciones en las dos direcciones perpendiculares en el plano del muro.

En lo que sigue se considera inicialmente que los ladrillos, comparativamente al mortero de pega, son infinitamente rígidos a todas las acciones: flexión, torsión, cortante y carga axial. Más adelante esta hipótesis se levantará aplicando un procedimiento aproximado.

El mecanismo que se considera para transmisión de cargas perpendiculares al plano del muro, cerca al borde libre y en la sección de máximo momento flector, transmite los esfuerzos internos parcialmente por torsión entre los ladrillos que forman la traba, como se muestra en la fig. 3, que muestra la sección de la fig. 2 en planta, y luego de presentarse la deformación medida por el ángulo de rotación $\theta$.

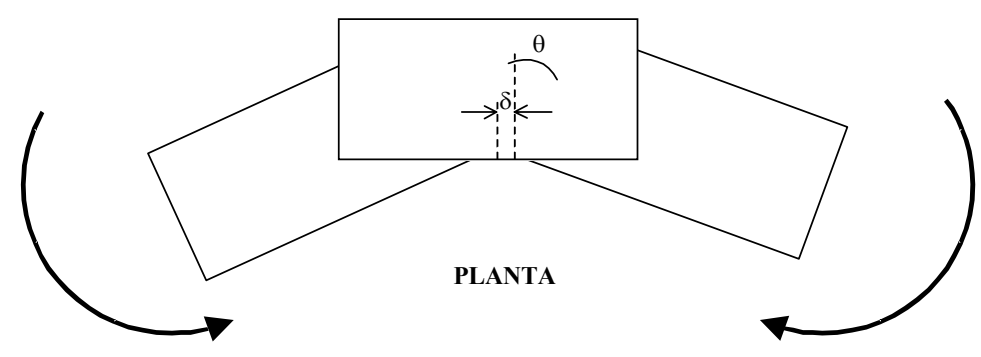

Figura 3. Mecanismo de transferencia de flexión a través de la traba entre ladrillos.

Los esfuerzos máximos de cortante inducidos por la torsión en el mortero de pega horizontal serían:

$$
\tau_{\max }=\frac{\theta}{b} \cdot G_{M T} \cdot \frac{t}{2} \cdot k
$$

donde:

$G_{M T}$ : Módulo de cortante del mortero cuando la acción es torsión

t/2: Distancia entre el centro de rotación y el borde del mortero

$k$ : $\quad$ Constante de proporcionalidad a definir en función de la sección transversal en consideración y del agrietamiento del mortero de pega horizontal.

Esta constante $k$ permite calcular el esfuerzo de corte sobre el mortero de pega horizontal y en la dirección $Y$, que es también la dirección en que se presenta el cortante por efecto del mecanismo de compresión del mortero de pega vertical que se describe más adelante. Es en esta dirección $Y$, independientemente que sea o no la dirección en donde se presenta el cortante máximo debido sólo a la torsión, en donde, por efecto de la suma del cortante por compresión del mortero de pega vertical, se presenta el cortante máximo final que define el estado límite del mecanismo propuesto. 
Una vez que la sección transversal gira el mortero de pega vertical se comprime, generando otra fuente adicional de resistencia a la flexión a la ya mencionada de la torsión habilitada por la traba de los ladrillos.

Debido a que la resistencia a la tracción del mortero de pega vertical es mucho menor que su resistencia a la compresión, necesariamente, aparte de la rotación $\theta$, se presenta un deslizamiento en dirección $Y$ de los ladrillos, $\delta$, que permite que se equilibren las fuerzas axiales sobre el ladrillo que está girando.

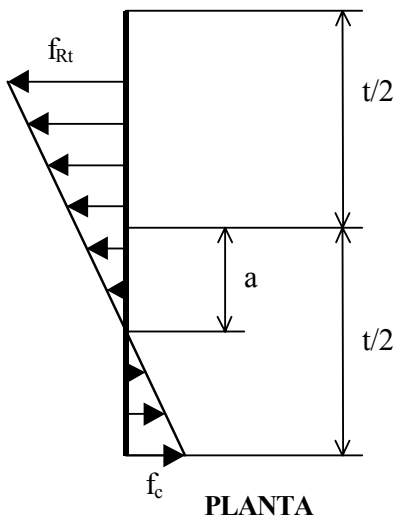

Figura 4. Distribución de esfuerzos sobre la cara del ladrillo en contacto con el mortero de pega vertical.

En la fig. 4 se muestra la distribución de esfuerzos sobre el ladrillo en la cara que está en contacto con el mortero de pega vertical. Como ya se mencionó, esta distribución se da luego de presentarse la rotación $\theta$ y el desplazamiento $\delta$ del ladrillo.

En la fig. $4, f_{R t}$ representa la resistencia a tracción del mortero de pega y $f_{c}$ representa el esfuerzo de compresión máximo luego de presentarse la rotación $\theta$ y el desplazamiento $\delta$. Si se acepta un comportamiento elástico del mortero se deriva la siguiente expresión:

$$
f_{c}=\frac{E_{M}}{h}(\theta \cdot t-2 \delta)
$$

donde:

$E_{M}: \quad$ Módulo de elasticidad del mortero.

La distancia $a$ que se muestra en la fig. 4 debe ser tal que se igualen los esfuerzos por compresión debidos a la rotación $\theta$ y los esfuerzos de tracción debidos al desplazamiento $\delta$. Si se llama:

$\eta=\frac{\delta}{\theta \cdot t}$ 
entonces:

$a=\eta \cdot t$

Además de los esfuerzos en el ladrillo en la cara que está en contacto con el mortero de pega vertical, se presentan esfuerzos en las caras que están en contacto con los morteros de pega horizontales, superior e inferior, debidos al cortante que se genera por el desplazamiento $\delta$ del ladrillo. La distribución de estos esfuerzos en ambas caras del ladrillo es la que se propone en la fig. 5.

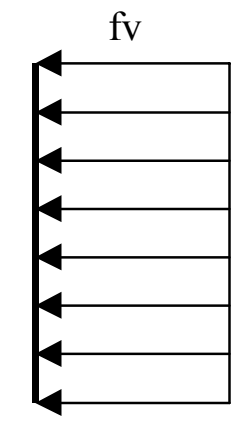

Figura 5. Distribución de esfuerzos de cortante en las caras superior e inferior del ladrillo donde el esfuerzo $f_{v}$ sobre el ladrillo está dado por:

$$
f_{v}=\frac{\delta}{b} \cdot G_{M s}
$$

En esta expresión:

$G_{M s}:$ Módulo de cortante del mortero cuando la acción es cortante pura

Para satisfacer el equilibrio de fuerzas en la dirección $Y$ del muro (figs. 1 y 2), es necesario que:

$$
\frac{f_{c}}{2}\left(\frac{t}{2}-a\right) w=f_{v} \cdot t \cdot(l-h)+\frac{(t / 2-a)}{f_{c}} \cdot \frac{f_{R t}^{2}}{2} w
$$

En esta ecuación se considera un cortante $f_{v}$ sobre el ladrillo en ambas caras, que es el caso que se ilustra en la fig. 2 y que corresponde a las hileras interiores de ladrillos del muro que se ilustra en la fig. 1. Si se considera el equilibrio de la última hilera de ladrillos del muro de la fig. 1, debe tenerse en cuenta el cortante desarrollado sobre una sola de las caras del ladrillo, resultando en:

$$
\frac{f_{c}}{2}\left(\frac{t}{2}-a\right) w=f_{v} \cdot t \cdot \frac{(l-h)}{2}+\frac{(t / 2-a)}{f_{c}} \cdot \frac{f_{R t}^{2}}{2} w
$$


Reemplazando en las ecs. 6 y 7 las ecs. 2, 3, 4 y 5 y considerando además que:

$$
f_{R t}=\frac{\delta \cdot E_{M}}{(h / 2)} \cdot \alpha
$$

es decir, que la resistencia a tracción del mortero, $f_{R t}$, es $\alpha$ veces las tracciones que generaría el desplazamiento $\delta$ del ladrillo, y además, nombrando la relación de aspecto del ladrillo, $r_{b}, \mathrm{y}$ de los morteros de pega vertical y horizontal, $r_{m}$, y la relación de módulos del mortero, $r_{G}$ :

$$
r_{b}=\frac{l-h}{w}, \quad r_{m}=\frac{h}{b}, \quad r_{G}=\frac{G_{M s}}{E_{M}}
$$

las ecs. de equilibrio 6 y 7 se pueden escribir como:

$4 \eta^{2}\left(1-\alpha^{2}\right)-4 \eta\left(1+r_{G} \cdot r_{m} \cdot r_{b}\right)+1=0$

$4 \eta^{2}\left(1-\alpha^{2}\right)-2 \eta\left(2+r_{G} \cdot r_{m} \cdot r_{b}\right)+1=0$

para hileras interiores de ladrillo en muros soportados en los lados inferior y superior, y para la última hilera de ladrillos de un muro con el lado superior libre, respectivamente.

Para seleccionar la raíz adecuada de estas ecuaciones se debe tener en cuenta la ec. 4 y la desigualdad $a<t / 2$, evidente de la fig. 4, que resulta en el siguiente límite:

$\eta \leq 0.5$

De acuerdo con lo expuesto, el momento resistente en dirección $Z, M_{R}$, del muro de mampostería (figs. 1 y 2), se puede expresar como:

$M_{R}=M_{R T}+M_{R C}$

donde $M_{R T}$ es la parte del momento resistente debido al mecanismo de torsión, y $M_{R C}$ la parte correspondiente al mecanismo de compresión del mortero de pega vertical. Estas dos componentes están dadas por las siguientes expresiones:

$$
M_{R T}=2 \frac{\theta}{b} G_{M T} \cdot K_{T}
$$

en el caso de las hileras interiores de ladrillo en muros soportados en los lados inferior y superior, $\mathrm{y}$

$$
M_{R T}=\frac{\theta}{b} G_{M T} \cdot K_{T}
$$


para la última hilera de ladrillos de un muro con el lado superior libre.

En las ecs. 14 y 15, $K_{T}$ representa la rigidez torsional de la sección del mortero de pega involucrada en el mecanismo de torsión. En los ejemplos que se ilustran más adelante se considera que esta sección transversal es rectangular de dimensiones $(l-h) / 2 \times$ x

El momento resistente debido al mecanismo de compresión del mortero de pega vertical, independientemente de si se trata de hileras interiores de un muro o de la última hilera de un muro libre en su extremo superior, considerando las ecs. 2, 3, 4, 5 y 8, está dado por:

$$
M_{R C}=\frac{E_{M} \cdot \theta \cdot t^{3} \cdot w}{12 h}\left(4 \eta^{3}\left(1+\alpha^{2}(2 \alpha-3)\right)-3 \eta+1\right)
$$

Los esfuerzos máximos de cortante inducidos en el mortero de pega horizontal, considerando esta vez los producidos por la torsión, $\theta$, y los producidos por el desplazamiento, $\delta$, resultan ser:

$$
\tau_{\max }=\frac{\theta \cdot t}{2 b}\left(G_{M t} \cdot k+2 G_{M s} \cdot \eta\right)
$$

Como el interés fundamental de este modelo está orientado a la evaluación de la aceleración horizontal perpendicular al plano del muro, $a_{\mathrm{R}}$, que es capaz de resistir un muro como el de la fig. 1, a continuación se calcula ésta. En lo que sigue se considera que el muro se encuentra simplemente apoyado en los muros laterales (fig. 1), que el claro libre es igual a $L$ y que el peso específico del muro de mampostería es $\gamma$. Si además, se considera que el momento flexionante máximo, $M_{\max }$, se presenta en el centro del claro y es igual al momento resistente, $M_{R}$, se tiene:

$$
\frac{a_{R}}{g}=\frac{8 M_{R}}{\gamma \cdot t \cdot(w+b) \cdot L^{2}}
$$

Como se mencionó inicialmente, en todo lo anterior se consideró que los ladrillos son infinitamente rígidos a todas las acciones: flexión, torsión, cortante, axial. Para considerar de manera simple la flexibilidad de los ladrillos se propone trabajar con módulos de elasticidad equivalentes, $G_{M T}{ }^{*}$ y $G_{M s}{ }^{*}$, que consideran la flexibilidad de los ladrillos, así:

Si se considera el mecanismo de torsión se tiene:

$$
G_{M T}^{*}=\frac{b}{K_{T}\left(\frac{l}{2 E_{b} I_{Z b}}+\frac{b}{G_{M t} K_{T}}\right)}
$$

donde: 
$E_{b}: \quad$ Módulo de elasticidad del ladrillo

$I_{z b}$ : Inercia del ladrillo alrededor del eje Z

Si se considera el mecanismo de compresión del mortero de pega vertical se tiene:

$$
G_{M s}^{*}=\frac{b}{A_{s Z M}\left(\frac{l}{2 E_{b} A_{Y b}}+\frac{b}{G_{M s} A_{s Z M}}\right)}
$$

donde:

\section{$E_{b}: \quad$ Módulo de elasticidad del ladrillo \\ $A_{Y b}:$ Área del ladrillo en la sección Y}

Las ecs. 19 y 20 permiten estimar los módulos de cortante equivalentes cuando las acciones son torsión y cortante pura, respectivamente, en función de los módulos elástico y de cortante del mortero y del ladrillo, y de las propiedades geométricas de estos dos elementos.

En el modelo propuesto se supone una resistencia infinita de los ladrillos a flexión; el estado límite de resistencia propuesto hasta el momento está determinado por el esfuerzo resistente máximo a cortante del mortero de pega horizontal, $\tau_{\max }$.

El momento flexionante resistente de los ladrillos, $M_{R b}$, se escribe en términos de la resistencia a la tracción de estos, $\sigma_{\operatorname{maxb}}$, como:

$$
M_{R b}=\frac{2 \cdot \sigma_{\operatorname{maxb}} \cdot I_{Z b}}{t}
$$

En definitiva, el momento resistente del muro está dado por el menor del estimado para los ladrillos, $M_{R b}$ (ec. 21), y la suma de los estimados para el mecanismo de torsión, $M_{R T}$ (ecs. 14 y 15), y el mecanismo de compresión del mortero de pega vertical, $M_{R C}$ (ec. 16).

La aceleración resistente, $a_{R}$, depende de manera muy importante de la longitud libre del muro, $L$; esta dimensión, al cuadrado, es inversamente proporcional a la aceleración resistente (ec. 18). Es posible mostrar también que hay una relación aproximadamente directa de la aceleración resistente con el espesor del muro $t$. Si la resistencia del muro está controlada por la resistencia a flexión de los ladrillos, esta relación es evidente de la ec. 21 si se tiene en cuenta que la inercia del ladrillo alrededor del eje $Z, I_{z b}$, es función del cubo del espesor del muro $t$. Cuando la resistencia del muro está controlada por los mecanismos de torsión y compresión propuestos, la relación directa entre el espesor del muro y la aceleración resistente se deduce de las ecs. 14, 15 y 16 si en las dos primeras se tiene en cuenta que la rigidez torsional del mortero de pega, $K_{T}$, es función del cubo del espesor del muro, y además, de la ec. 17 se deduce que el giro $\theta$ es inversamente proporcional a $t$. Todo lo anterior reemplazado en la ec. 18 muestra la relación propuesta entre $t$ y $a_{R}$. 
Igualmente, es posible mostrar que la longitud del ladrillo, $l$, es aproximadamente proporcional al momento resistente de la sección, $M_{R}$, y debido a que esta dimensión no afecta la masa del muro, finalmente se mantiene la misma proporcionalidad directa entre $l$ y la aceleración resistente del muro. Lo anterior es válido si es el mecanismo de torsión y compresión propuesto el que controla la resistencia del muro. Si esta última es controlada por la resistencia a flexión de los ladrillos, la longitud del ladrillo es independiente de la aceleración resistente del muro, lo que define un límite a la longitud del ladrillo. Si se supone que inicialmente la resistencia del muro está controlada por el mecanismo de torsión y compresión propuesto, esta se puede aumentar fácilmente incrementando la longitud de los ladrillos. Esta estrategia es válida hasta que se alcanza la resistencia proporcionada por éstos, y a partir de este momento incrementar la longitud de los ladrillos no aumenta la resistencia del muro, lo que hace inútil la acción y señala un límite a la longitud óptima de los ladrillos para que se alcancen al mismo tiempo los dos mecanismos de resistencia.

En el caso de la altura de los ladrillos, $w$, es posible mostrar que si la resistencia del muro está controlada por el mecanismo de torsión y compresión propuesto, esta dimensión no afecta apreciablemente la resistencia de la sección, pero como aumenta de manera directa la masa de la sección resistente, resulta finalmente una proporcionalidad inversa entre $w$ y la aceleración resistente. Este hecho, como en el caso anterior, señala un límite sobre el espesor mínimo de los ladrillos para que se alcancen al mismo tiempo los dos mecanismos de resistencia.

Igualmente, es posible mostrar que la relación de los espesores de los morteros de pega, $b$ y $h$ con el momento resistente de la sección, $M_{R}$, y con la aceleración resistente de la sección, $a_{R}$, es inversa pero no proporcional como en los casos anteriores.

Ni los módulos de elasticidad de los materiales ni las pequeñas resistencias a la tracción de los morteros de pega, $f_{R t}$, influyen de manera importante ni en el momento resistente de la sección ni en la aceleración resistente de ésta. En particular, en referencia a $f_{R t}$, trabajar con un valor de $\alpha$ igual a cero (ec. 10) no modifica apreciablemente la resistencia que se calcularía haciendo uso de la resistencia a la tracción del mortero de pega.

Si se quisiera aumentar la aceleración resistente de un muro de mampostería no reforzada, se deberían controlar con cuidado las variables mencionadas en los párrafos anteriores, en especial, se debería disminuir la longitud libre del muro, $L$, y aumentar el espesor del muro, $t$. La longitud de los ladrillos, $l$, debería aumentarse hasta un límite en que la resistencia sea controlada por los ladrillos. Igualmente, se debe disminuir la altura de los ladrillos hasta un límite en que la resistencia sea controlada por estos últimos. En menor proporción, se deberían mantener espesores de pega lo más pequeño posibles.

\section{EJEMPLOS DE APLICACIÓN}

A continuación se presentan los cálculos y resultados de la aplicación del modelo analítico a dos casos, que como más adelante se detallará, se ensayaron a escala natural. 


\section{Muro 1}

Se estudiará en este caso un muro de mampostería no reforzada hecho a base de bloques de concreto macizo. Las dimensiones del muro son las que a continuación se detallan (fig. 6):

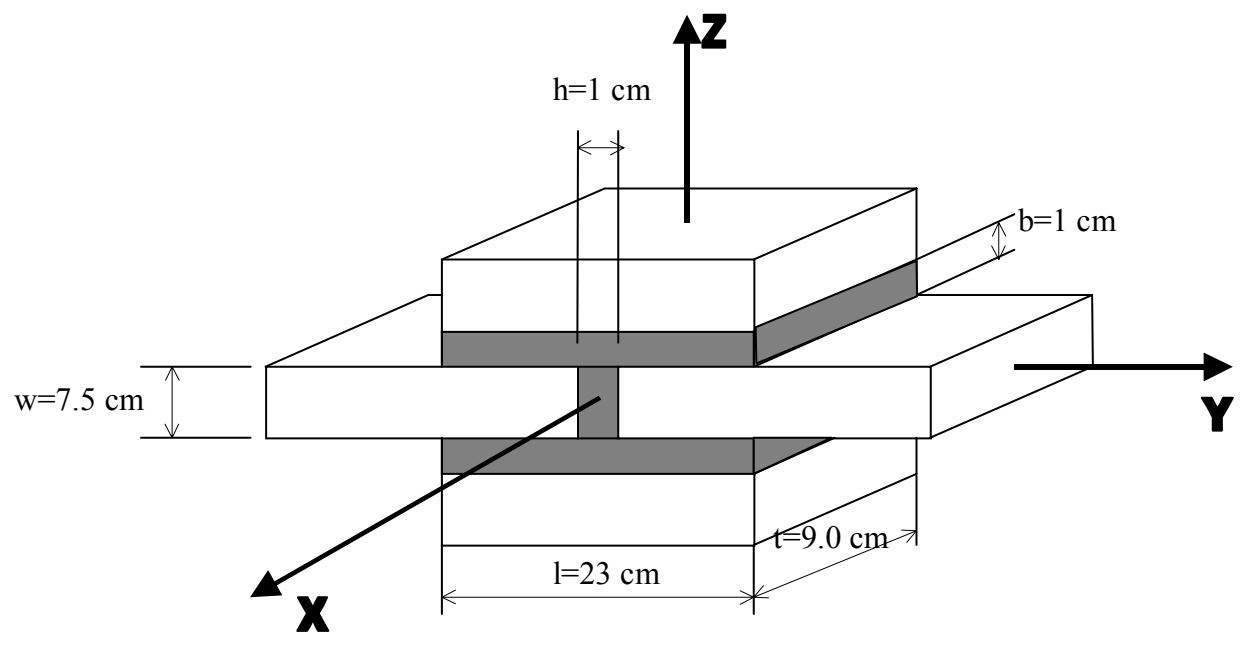

Figura 6. Dimensiones del Muro 1

Las propiedades geométricas de las secciones son las que a continuación se indican:

$$
\begin{aligned}
A_{Y b} & =t \cdot w=67.5 \mathrm{~cm}^{2} & A_{s Z M}=\frac{l-h}{2} \cdot t \cdot \frac{1}{1.2}=82.5 \mathrm{~cm}^{2} \\
I_{Z b} & =\frac{t^{3} \cdot w}{12}=455.63 \mathrm{~cm}^{4} & K_{T}=\frac{l-h}{2} \cdot t^{3} \cdot \frac{1}{6.2}=1293.39 \mathrm{~cm}^{4} \\
k & =2 \cdot \frac{4.60}{6.20}=1.48 &
\end{aligned}
$$

Para el cálculo de $k$ y $K_{T}$ se consideró que la sección transversal de mortero de pega sometida a torsión es rectangular de dimensiones $(l-h) / 2 \times \mathrm{x}$, y que la rigidez torsional y el factor para cálculo de esfuerzos máximos, $k$, son las que corresponden a una sección rectangular de las dimensiones indicadas (ver por ejemplo Timoshenko y Goodier, 1970).

Las relaciones de aspecto de los ladrillos, de las pegas y modulares, son las que se indican a continuación:

$$
r_{m}=1.0, \quad r_{b}=2.93 \quad r_{G}=0.07
$$

Para este muro se consideran las siguientes propiedades de los materiales:

$$
\begin{aligned}
& E_{b}=1.2 \mathrm{E} 04 \mathrm{MPa} \\
& G_{b}=5.4 \mathrm{E} 03 \mathrm{MPa} \\
& E_{M}=1.0 \mathrm{E} 04 \mathrm{MPa} \\
& G_{M}=4.0 \mathrm{E} 03 \mathrm{MPa}
\end{aligned}
$$


$\tau_{\max },=0.5 \mathrm{MPa}$

Los módulos de cortante equivalentes del mortero de pega son los siguientes:

$G_{M T}{ }^{*}=336.65 \mathrm{MPa} \quad G_{M s}{ }^{*}=703.58 \mathrm{MPa}$

Si se considera nula la resistencia a tracción del mortero de pega, esto es: $\alpha=0.0$, la ec. cuadrática 11 en términos de $\eta$ resulta igual a:

$$
\begin{aligned}
& 4 \eta^{2}-4.82 \eta+1.0=0 \\
& \rightarrow \eta=0.27
\end{aligned}
$$

Reemplazando este valor de $\eta$, correspondiente a borde libre, en la ec. 17, se encuentra la rotación máxima, $\theta$, que se presentaría en este muro en la última hilera libre de ladrillos: $\theta=$ 1.173 E-04 rad. Finalmente, reemplazando en las ecs. 15 y 16 los valores hasta ahora conseguidos se obtienen los momentos resistentes por el mecanismo de torsión y de compresión del mortero de pega vertical, respectivamente, para el caso de borde libre.

$$
\begin{aligned}
& M_{R T}=51 \mathrm{~N} \cdot \mathrm{m} \\
& M_{R C}=91 \mathrm{~N} \cdot \mathrm{m}
\end{aligned}
$$

El momento resistente total resulta ser igual a:

$$
M_{R}=142 \mathrm{~N} \cdot \mathrm{m}
$$

y de este, el $36 \%$ se debe al mecanismo de torsión y el restante $64 \%$ se debe al mecanismo de compresión sobre el mortero de pega vertical.

Si se considera que la resistencia a la tracción máxima de los ladrillos es $3.0 \mathrm{MPa}$, el momento resistente de los ladrillos resulta ser igual a $304 \mathrm{~N} \cdot \mathrm{m}$, lo que significa que el momento resistente del muro es igual a $142 \mathrm{~N} \cdot \mathrm{m}$.

A continuación se calcula la aceleración horizontal, $a_{R}$, que sería capaz de soportar un muro como el que se muestra en la fig. 1 hecho con los materiales propuestos. Se proponen los siguientes dos parámetros adicionales que permiten estimar la aceleración horizontal resistente: la longitud horizontal libre, $L$, igual a $2.80 \mathrm{~m}$, y el peso específico del muro, $\gamma$, igual a $20 \mathrm{KN} / \mathrm{m}^{3}$.

Reemplazando estos valores y los anteriormente conseguidos en la ec. 18, se obtiene:

$$
\frac{a_{R}}{g}=0.95
$$

Más adelante se compararán estos resultados con un ensayo a escala natural en mesa vibradora de este muro. 


\section{Muro 2}

Se estudiará en este caso un muro de mampostería no reforzada con ladrillos de arcilla cocida. Las dimensiones del muro son las que a continuación se detallan (fig. 7):

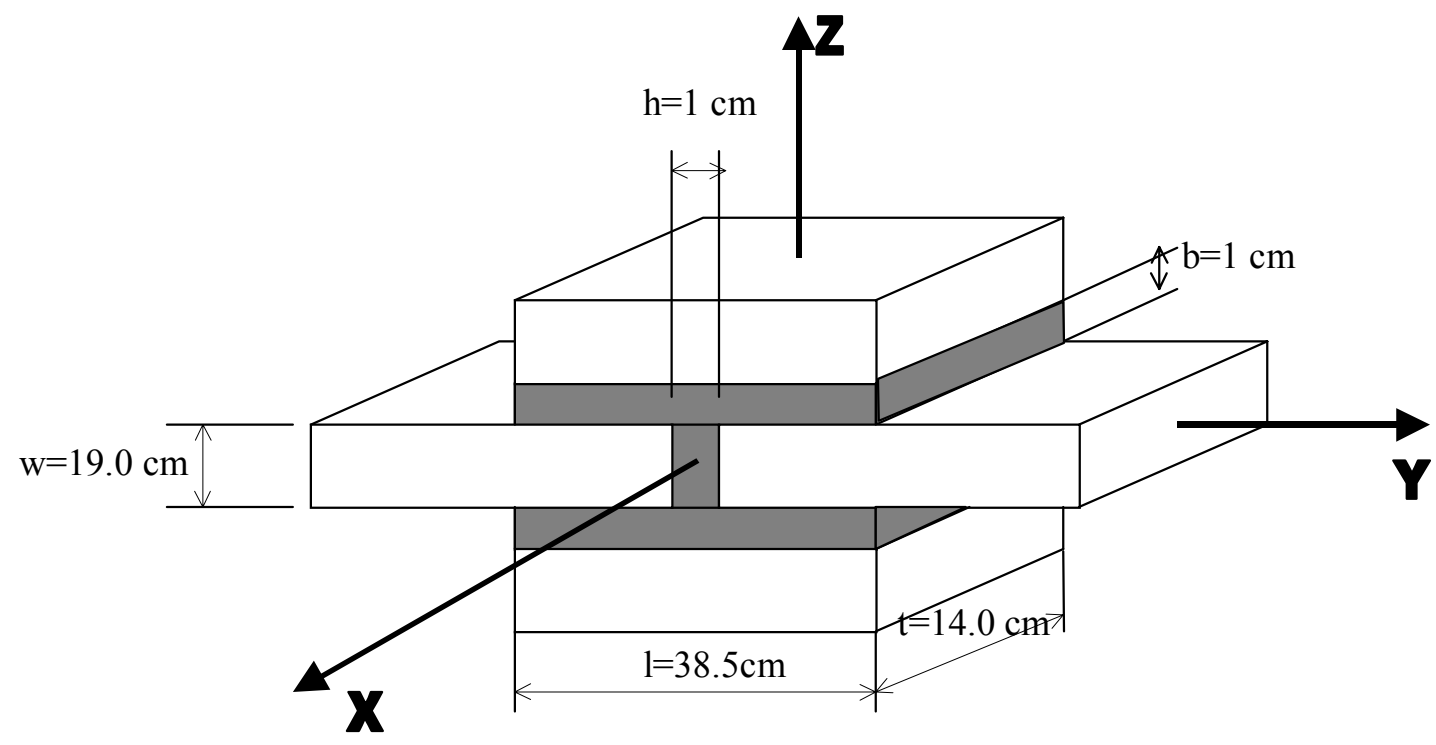

Figura 7. Dimensiones del Muro 2

Las propiedades geométricas de las secciones son las que a continuación se indican. Para considerar que la sección del ladrillo es hueca, en el cálculo de las propiedades de éste se usará un factor de reducción del área de 0.5 y un factor de reducción del momento de inercia de 0.75.

$$
\begin{aligned}
A_{Y b} & =t \cdot w \cdot 0.5=133.0 \mathrm{~cm}^{2} & A_{s Z M}=\frac{l-h}{2} \cdot t \cdot \frac{1}{1.2}=218.75 \mathrm{~cm}^{2} \\
I_{Z b} & =\frac{t^{3} \cdot w}{12} \cdot 0.75=3258.50 \mathrm{~cm}^{4} & K_{T}=\frac{l-h}{2} \cdot t^{3} \cdot \frac{1}{6.2}=8298.39 \mathrm{~cm}^{4} \\
k & =2 \cdot \frac{4.60}{6.20}=1.48 &
\end{aligned}
$$

Las relaciones de aspecto de los ladrillos, de las pegas y modulares, son las que a continuación se indican:

$$
r_{m}=1.0 \quad r_{b}=1.97 \quad r_{G}=0.0346
$$

Para este muro se consideran las siguientes propiedades de los materiales:

$$
\begin{aligned}
& E_{b}=1.2 \mathrm{E} 04 \mathrm{MPa} \\
& G_{b}=5.4 \text { E } 03 \mathrm{MPa} \\
& E_{M}=1.0 \mathrm{E} 04 \mathrm{MPa}
\end{aligned}
$$




$$
\begin{aligned}
& G_{M}=4.0 \mathrm{E} 03 \mathrm{MPa} \\
& \tau_{\max }=0.4 \mathrm{MPa}
\end{aligned}
$$

Los módulos de cortante equivalentes del mortero de pega son los siguientes:

$$
G_{M T}^{*}=230.66 \mathrm{MPa} \quad G_{M s} *=346.21 \mathrm{MPa}
$$

Si se considera nula la resistencia a tracción del mortero de pega, esto es, $\alpha=0.0$, la ec. cuadrática 11 en términos de $\eta$ resulta igual a:

$$
\begin{aligned}
& 4 \eta^{2}-4.14 \eta+1.0=0 \\
& \rightarrow \eta=0.38
\end{aligned}
$$

Reemplazando este valor de $\eta$, correspondiente a borde libre, en la ec. 17, se encuentra la rotación máxima, $\theta$, que se presentaría en este muro en la última hilera libre de ladrillos: $\theta=$ 9.405 E-05 rad. Finalmente, reemplazando en las ecs. 15 y 16 los valores hasta ahora conseguidos, se obtienen los momentos resistentes por el mecanismo de torsión y de compresión del mortero de pega vertical, respectivamente, para el caso de borde libre:

$$
\begin{aligned}
& M_{R T}=181 \mathrm{~N} \cdot \mathrm{m} \\
& M_{R C}=327 \mathrm{~N} \cdot \mathrm{m}
\end{aligned}
$$

El momento resistente total resulta ser igual a:

$$
M_{R}=508 \mathrm{~N} \cdot \mathrm{m}
$$

y de este, el $36 \%$ se debe al mecanismo de torsión, y el restante $64 \%$ se debe al mecanismo de compresión sobre el mortero de pega vertical.

Si se considera que la resistencia a la tracción máxima de los ladrillos es 3.0 MPa, el momento resistente de los ladrillos resulta ser igual a $1397 \mathrm{~N} \cdot \mathrm{m}$, lo que significa que el momento resistente del muro es igual a $508 \mathrm{~N} \cdot \mathrm{m}$.

A continuación se calcula la aceleración horizontal, $a_{R}$, que sería capaz de soportar un muro como el que se muestra en la fig. 1 hecho con los materiales propuestos. Se proponen los siguientes dos parámetros adicionales que permiten estimar la aceleración horizontal resistente: la longitud horizontal libre, $L$, igual a $2.80 \mathrm{~m}$, y el peso específico del muro, $\gamma$, igual a $11 \mathrm{KN} / \mathrm{m}^{3}$

Reemplazando estos valores y los anteriormente conseguidos en la ec. 17, se obtiene:

$$
\frac{a_{R}}{g}=1.68
$$


Más adelante se compararán estos resultados con un ensayo a escala natural en mesa vibradora de este muro.

\section{ENSAYOS}

Para verificar el mecanismo de transmisión de cargas propuesto y las aceleraciones resistentes calculadas, se sometieron a ensayo dos muros exactamente iguales a los de los ejemplos propuestos anteriormente (fig. 8). Estos ensayos se ejecutaron en la mesa vibradora del laboratorio de estructuras de la Universidad de Canterbury en Nueva Zelanda. A continuación se darán algunos detalles de los ensayos.

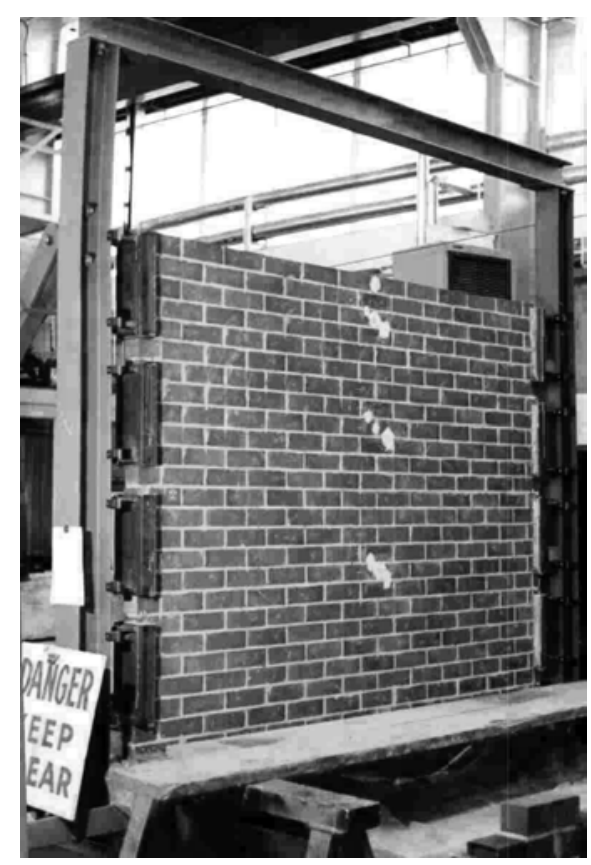

Figura 8. Marco de prueba y muro de mampostería no reforzada

\section{Marco de prueba.}

La conexión en la base de los muros se hizo como tradicionalmente se hace: fijándolos a la cimentación con un mortero de las mismas características del mortero de pega del muro. Para simular la articulación en los extremos laterales del muro, como se supone en el modelo que permite calcular la aceleración resistente del muro, se construyó un marco en acero con articulaciones de $40 \mathrm{~cm}$ de longitud como muestra la fig. 8. Estas articulaciones permiten que la rotación en los extremos cambien con la altura, de un máximo en la parte superior del muro, completamente libre, a una rotación nula en la base, completamente ligada a la cimentación. Igualmente, las conexiones en los extremos laterales permiten el desplazamiento en dirección Y del muro (fig. 1), evitando la transmisión de cargas en esta dirección por efecto de arco. 


\section{Excitación}

Para excitar el muro se utilizó una señal armónica con un periodo de $1.0 \mathrm{~s}$ en el primer muro y de $0.8 \mathrm{~s}$ en el segundo muro. El cambio obedeció a la necesidad de aumentar la aceleración en el segundo caso y a restricciones en el desplazamiento máximo del dispositivo de aplicación del movimiento armónico.

Los periodos tan altos del movimiento armónico se seleccionaron para evitar al máximo la resonancia con el periodo propio de vibración del muro, cercano a $0.1 \mathrm{~s}$. En estas condiciones, el espectro de respuesta de aceleraciones de la excitación es prácticamente plano en periodos cercanos al de la estructura, lo que elimina las incertidumbres al momento de estimar la aceleración espectral actuando sobre el muro: prácticamente igual a la aceleración máxima del movimiento armónico de excitación.

La amplitud de la aceleración aplicada se incrementó cada $0.05 \mathrm{~g}$ empezando en $0.30 \mathrm{~g}$ y hasta alcanzar la falla del espécimen, en caso de alcanzarla.

El tiempo de duración de la excitación fue de 30 segundos en el primer muro y de 60 segundos en el muro 2, con rampas de ascenso y descenso de 5 segundos al principio y al final de la excitación, respectivamente. El cambio en la duración obedeció al hecho de que se detectó que en el primer caso los 30 segundos no eran suficientes para alcanzar la amplitud máxima deseada en la base del muro: durante estos, las amplitudes siempre fueron crecientes y no apareció una tendencia asintótica que mostrara que se estaba alcanzando la amplitud máxima del movimiento en la base. Todo lo anterior debido exclusivamente al dispositivo de aplicación del movimiento armónico.

\section{Instrumentación}

Para efectos de verificar el modelo planteado se instalaron sensores extensométricos, acelerómetros y medidores de desplazamiento para medir: deformaciones, aceleraciones y desplazamientos en el muro. Las figs. 9 y 10 muestran la disposición de la instrumentación dentro de los muros 1 y 2 , respectivamente.

Para leer los sensores extensométricos ubicados en los morteros de pega vertical y horizontal se utilizó un canal del sistema de captura de datos para cada uno de los lados del muro; frontal y posterior. Lo anterior debido a que en estos sitios se esperaba una asimetría grande en la historia de deformaciones producto del agrietamiento cuando se sometía la sección a tracción. Por el contrario, los sensores ubicados en las dos caras de los ladrillos se leyeron utilizando medio puente y un solo canal del sistema de captura de datos. En los ladrillos se esperaba una deformación simétrica en tracción y compresión. En todos los casos se usaron amplificadores de la señal debido a que se esperaban deformaciones muy pequeñas, del orden de $40 \mu \varepsilon$.

Para verificar qué porcentaje de la carga se transmitía en dirección horizontal, como lo postula el modelo propuesto, y qué porcentaje se transmitía en dirección vertical como se 
propone en los modelos que se basan en la transmisión de carga debido a la precompresión por carga axial, se utilizaron sensores tanto en las juntas verticales como horizontales en un arreglo que se repite en tres zonas del muro: en la parte superior, a $2 / 3$ de la altura y a $1 / 3$ de la altura, aproximadamente (figs. 9 y 10).


Figura 9. Disposición de la instrumentación dentro del muro 1
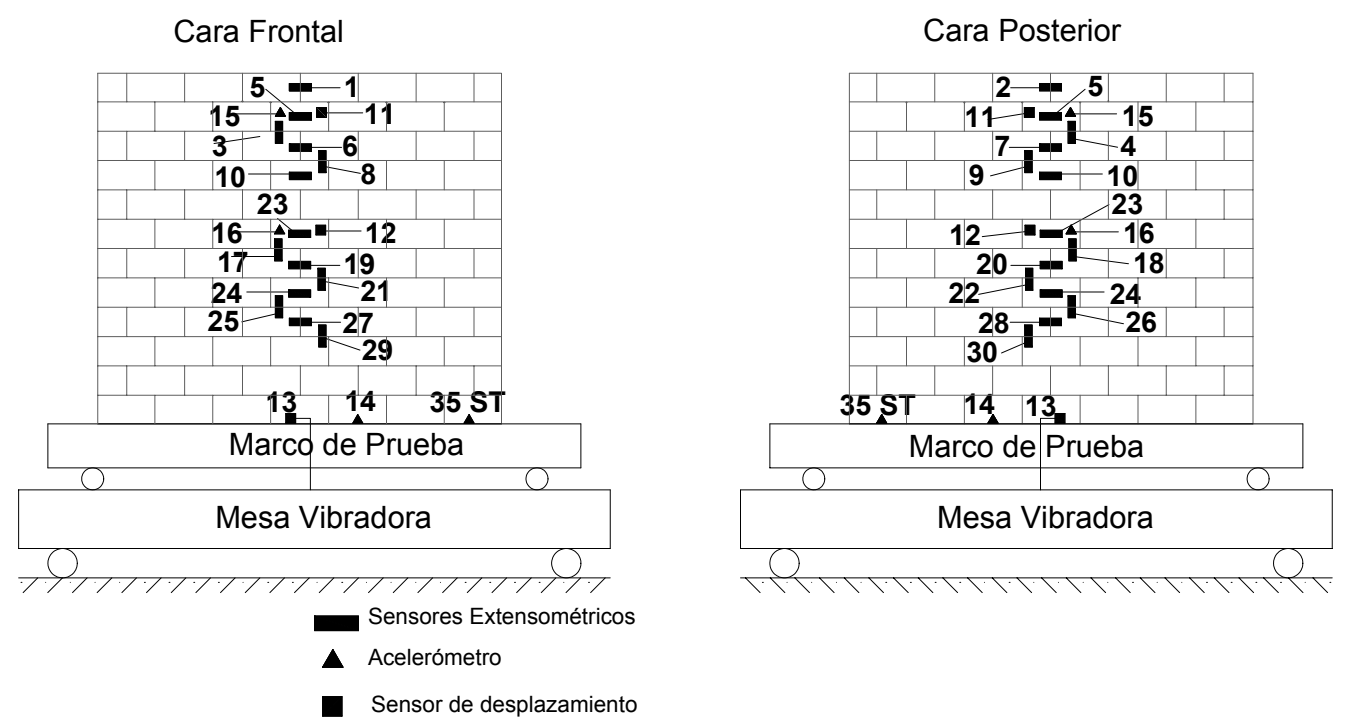

Figura 10. Disposición de la instrumentación dentro del muro 2

Los sensores de desplazamiento ubicados en el arreglo superior y a $2 / 3$ de la altura se fijaron al marco de prueba de manera que registraran el desplazamiento relativo del eje de simetría del muro respecto a sus extremos. 


\section{Probetas}

Adicionalmente a los ensayos sobre los muros a escala natural, se diseñó un ensayo sobre una muestra de muro semejante a la que se indica en la fig. 2, que indique el momento resistente del muro de acuerdo con el mecanismo de transmisión de cargas propuesto. En la fig. 11 se muestra el dispositivo usado durante el ensayo de una probeta. En la parte inferior de la foto se ilustra un tornillo sin fin que permite aplicar una carga perpendicular a dos brazos metálicos que la transmiten como una carga de compresión axial más un momento flector a la probeta que se ilustra en la parte superior de la foto. Debido a la gran longitud de los brazos metálicos, la carga axial que se transmite a la probeta es despreciable en comparación con el momento flector inducido.

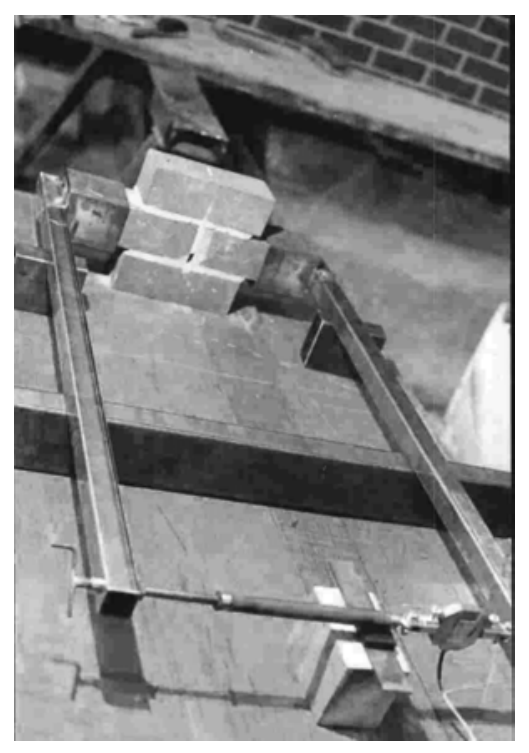

Figura 11. Ensayo de una probeta

Se alcanza a apreciar en la parte inferior de la foto (fig. 11) la celda de carga que mide la fuerza aplicada en los extremos de los brazos metálicos.

\section{RESULTADOS}

\section{Muro 1}

La aceleración máxima alcanzada en la base de este muro está entre $0.8 \mathrm{~g}$, la última prueba registrada y $0.9 \mathrm{~g}$, la aceleración a la que falló el muro. De los registros es clara una asimetría en la respuesta del sensor extensométrico 2 ubicado en la parte superior del muro, indicando con esto la fisuración del mortero de pega vertical por efecto de la tracción. Este registro es también el que alcanza una deformación mayor por tracción, $550 \mu \varepsilon$, y $150 \mu \varepsilon$ del lado de compresión. El registro del sensor extensométrico 7 ubicado en el mortero de pega vertical pero sobre la tercera 
hilera de ladrillos de arriba hacia abajo, muestra una respuesta perfectamente simétrica, indicando que no se alcanzó el límite de resistencia a la tracción del mortero de pega. En este caso se alcanzaron deformaciones del orden de $150 \mu \varepsilon$ en ambas direcciones. El registro del sensor extensométrico 20, ubicado sobre la novena hilera de ladrillos de arriba hacia abajo, tiene un comportamiento enteramente similar al de la tercera hilera de ladrillos (sensor 7). Finalmente, el sensor extensométrico 28, ubicado en el mortero de pega vertical pero sobre la $17^{\text {ava }}$ hilera de ladrillos de arriba hacia abajo, sigue mostrando un trabajo grande de transmisión de cargas en dirección horizontal, alcanzando tanto a tracción como a compresión deformaciones del orden de $100 \mu \varepsilon$ (fig. 12).
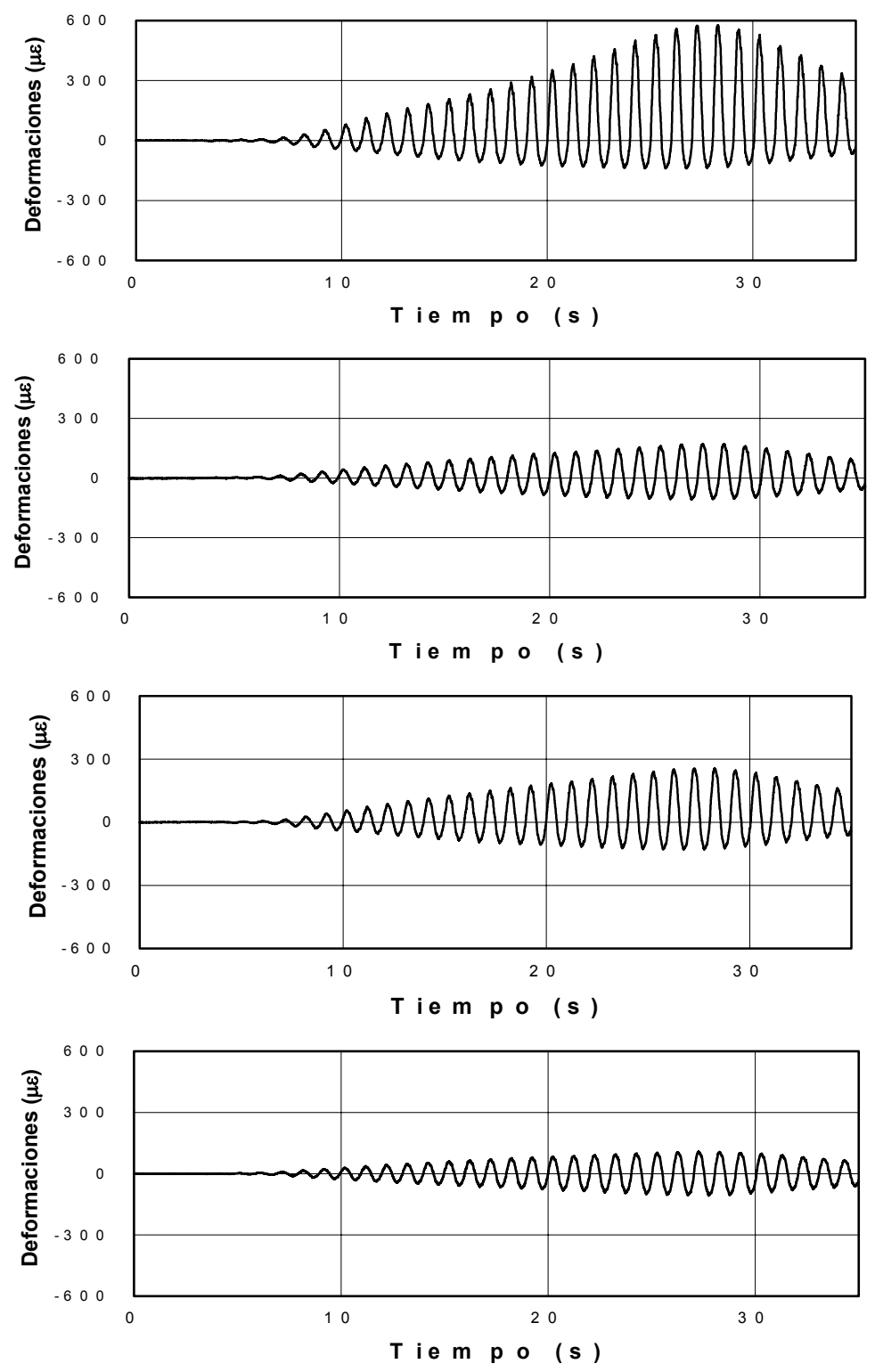

Figura 12. Registros de los sensores extensométricos 2, 7, 20 y 28 ubicados en los morteros de pega vertical cuando se alcanzó una aceleración de $0.8 \mathrm{~g}$ en el muro 1. 
Los registros de los sensores extensométricos 4, 18 y 30, ubicadas en los morteros de pega horizontal en la parte superior del muro, a los $2 / 3$ de la altura y a $1 / 3$ de la altura, respectivamente, indican claramente cómo se incrementa la transmisión de cargas en dirección vertical a medida que se acerca a la cimentación del muro, debido al aumento de la carga axial que actúa como precompresión del muro y posibilita la transmisión de momentos flexionantes. El registro del sensor 30 muestra alguna asimetría producto de la fisuración del mortero de pega por efecto de las tracciones sobre éste (fig. 13).
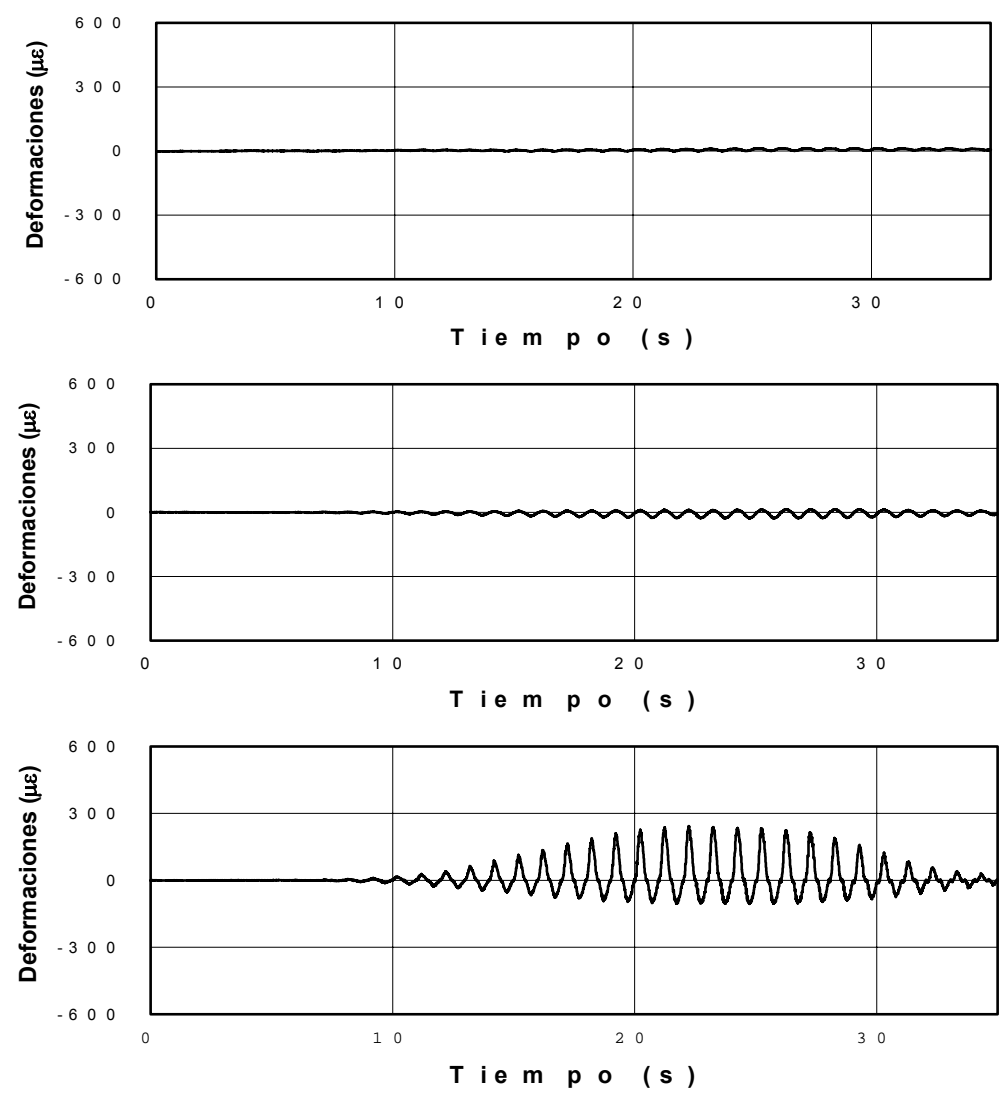

Figura 13. Registros de los sensores extensométricos 4, 18 y 30 ubicados en los morteros de pega horizontal cuando se alcanzó una aceleración de $0.8 \mathrm{~g}$ en el muro 1 .

Los registros del desplazamiento relativo del muro en las hileras de ladrillo 2 y 8 son muy similares, alcanzando en ambos casos un desplazamiento máximo relativo del orden de $3 \mathrm{~mm}$.

Como era de esperarse, los registros de las aceleraciones en el muro a nivel de las hileras de ladrillo 2 y 8 son muy similares, alcanzando una aceleración máxima de $0.8 \mathrm{~g}$.

Simultáneamente a la construcción de este muro se prepararon 27 probetas y se ensayaron el mismo día que se ensayó el muro. Los resultados son los siguientes: el momento resistente promedio resultó ser de $311 \mathrm{~N} \cdot \mathrm{m}$ con una desviación de $59 \mathrm{~N} \cdot \mathrm{m}$. Asombra la baja desviación encontrada, lo que habla muy bien del diseño de la prueba. 


\section{Muro 2}

La aceleración máxima alcanzada en la base del muro, por limitaciones del dispositivo de aplicación de carga, fue de $1.8 \mathrm{~g}$. Después de esto, se volteó el muro y se le aplicó carga gravitacional perpendicular al plano hasta la falla.

Los registros de los sensores extensométricos revelan un comportamiento enteramente similar al reportado para el muro 1: en la parte superior del muro prevalece la transmisión de cargas en dirección horizontal, pasando gradualmente a una transmisión de cargas en dirección vertical a medida que se acerca a la cimentación del muro, debido al aumento de carga axial que actúa como precompresión del muro y posibilita la transmisión de momentos flexionantes.

Este muro se ensayó a $0.4 \mathrm{~g}$ de aceleración en tres ocasiones: inicialmente cuando se llegó a este nivel de aceleración en el proceso ya descrito de incremento de la aceleración cada $0.05 \mathrm{~g}$. En una segunda ocasión justamente después del ensayo a $0.8 \mathrm{~g}$ de aceleración, y la tercera vez justamente después del ensayo a $1.0 \mathrm{~g}$ de aceleración. Se trataba de verificar con estos ensayos el efecto del agrietamiento del muro producido por una historia de altos niveles de aceleración.

De los registros obtenidos es evidente una acumulación del daño en la primera hilera del muro en términos de una pérdida de rigidez de la sección resistente: las deformaciones que se alcanzan para el mismo nivel de carga, $0.4 \mathrm{~g}$, son aproximadamente del doble cuando previamente se ha alcanzado una carga del doble, $0.8 \mathrm{~g}$, y aproximadamente de cinco veces cuando previamente se ha alcanzado una carga 2.5 veces más grande, $1.0 \mathrm{~g}$.

La acumulación de daño también es evidente en la tercera hilera de ladrillos, en este caso las deformaciones que se alcanzan para el mismo nivel de carga, $0.4 \mathrm{~g}$, son aproximadamente de 1.5 veces cuando previamente se ha alcanzado una carga del doble, $0.8 \mathrm{~g}$, y aproximadamente del doble, cuando previamente se ha alcanzado una carga 2.5 veces más grande, $1.0 \mathrm{~g}$.

Cuando no se alcanza el nivel de fisuración del mortero de pega vertical, lo que significa que no hay daño, se esperaría un comportamiento elástico, evidente en el comportamiento de las hileras de ladrillos séptima y novena.

Según el modelo propuesto, la rigidez de la sección es función de qué tanto ha penetrado la grieta de tracción en el mortero de pega vertical. La rigidez mínima se alcanza para una deformación en el punto de falla que es cuando la grieta de tracción penetra al máximo en el mortero de pega vertical. Un cálculo conservador de la rigidez de la sección se puede estimar con base en una resistencia a la tracción nula del mortero de pega vertical.

Debido a que este muro no se pudo llevar a la falla aplicando cargas inerciales producto de aceleraciones horizontales perpendiculares al plano del muro y a sabiendas de que se habían alcanzado niveles de aceleración horizontal del orden de $1.8 \mathrm{~g}$, se decidió rotar el muro $90^{\circ} \mathrm{y}$ someterlo a su peso propio, lo que equivale a $1.0 \mathrm{~g}$, y a partir de este momento comenzar a cargarlo uniformemente hasta producir la falla. 
El peso uniformemente distribuido que se le aplicó al muro para producir su falla fue el equivalente a una aceleración horizontal que está entre $1.43 \mathrm{~g}$ y $1.55 \mathrm{~g}$. Aunque esta carga es menor que la soportada previamente como aceleración horizontal, $1.8 \mathrm{~g}$, está cercana a esta última. Es posible que en el procedimiento de rotación del muro se haya producido algún daño a la estructura.

Finalmente, el patrón de grietas al momento de la falla sigue las líneas de rotura que se esperan de una placa con las condiciones de borde del muro (fig. 14). Es interesante notar que la grieta vertical que se inicia en la parte superior del muro atraviesa los ladrillos, significando con esto que en las hileras de ladrillos 2, 4 y 6 se alcanzó la resistencia de los ladrillos antes de agotar el mecanismo de torsión y compresión del mortero de pega vertical propuesto. La resistencia a flexión de los ladrillos son pues, como se prevé en el modelo, un límite de resistencia del muro que debe ser considerado.

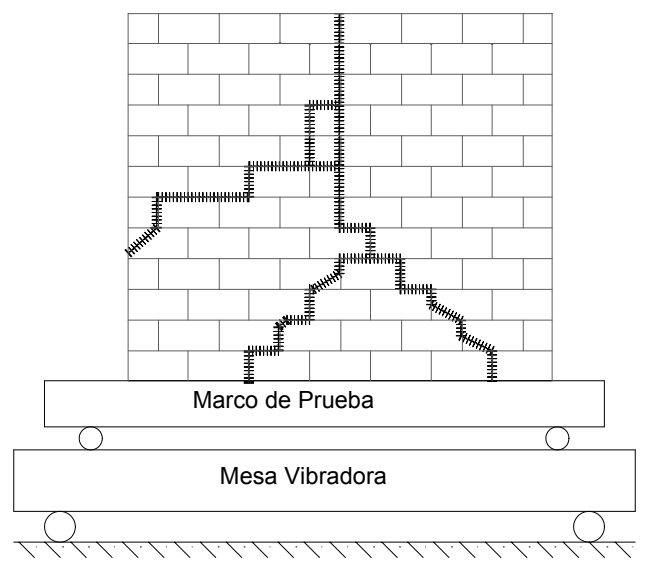

Figura 14. Patrón de agrietamiento del muro 2 al momento de la falla

Simultáneamente a la construcción del muro se prepararon 30 probetas, 15 con mortero de pega vertical y 15 sin mortero de pega vertical, para ser ensayadas con el dispositivo que ya se explicó. La idea era ver con el ensayo los porcentajes de participación de los dos mecanismos postulados: el de torsión y el de compresión del mortero de pega vertical.

Los ensayos a las probetas se ejecutaron la misma semana que se ensayó el muro a escala natural. Los resultados son los siguientes: El momento resistente promedio de las probetas con mortero de pega vertical resultó ser de $800 \mathrm{~N} \cdot \mathrm{m}$ con una desviación de $100 \mathrm{~N} \cdot \mathrm{m}$, y el momento resistente promedio de las probetas sin mortero de pega vertical resultó ser de $400 \mathrm{~N}$ m con una desviación de $50 \mathrm{~N} \cdot \mathrm{m}$. Los porcentajes de participación de los dos mecanismos según estos resultados son 50\% cada uno, que no están lejos de los postulados en el modelo analítico: 36\% para el mecanismo de torsión, y 64\% para el mecanismo de compresión de la junta vertical. Nuevamente, asombra la baja desviación encontrada, lo que confirma el buen diseño de la prueba. 


\section{ANÁLISIS DE RESULTADOS}

En el muro 1 la aceleración resistente, $a_{R}$, predicha por el modelo, $0.95 \mathrm{~g}$, está un poco por encima de la que finalmente se alcanzó en la prueba, aproximadamente $0.85 \mathrm{~g}$. Por el contrario, el momento resistente de las hileras interiores de ladrillo predicha por el modelo, $265 \mathrm{~N} \cdot \mathrm{m}$, está por debajo del promedio que resultó de la prueba de las probetas, $311 \mathrm{~N} \cdot \mathrm{m}$.

En el muro 2 la aceleración resistente, $a_{R}$, predicha por el modelo, $1.68 \mathrm{~g}$, está por debajo de la última que se logró registrar en el dispositivo de aplicación de cargas inerciales, $1.80 \mathrm{~g}$. Sin embargo, la aceleración equivalente máxima conseguida a través de la aplicación de cargas gravitacionales, entre $1.44 \mathrm{~g}$ y $1.53 \mathrm{~g}$, indican que posiblemente la aceleración resistente estaba muy cerca de la última aceleración lograda con cargas inerciales. En cuanto al momento resistente de las hileras interiores de ladrillo predicho por el modelo, $919 \mathrm{~N} \cdot \mathrm{m}$, está un poco por encima del promedio que resultó de la prueba de las probetas, $800 \mathrm{~N} \cdot \mathrm{m}$. Por el contrario, el momento resistente de las hileras interiores cuando solo se considera el mecanismo de torsión, $327 \mathrm{~N} \cdot \mathrm{m}$, está un poco por debajo del promedio que resultó de la prueba de las probetas sin mortero de pega vertical, $400 \mathrm{~N} \cdot \mathrm{m}$.

Aunque en algunos casos las desviaciones entre lo predicho por el modelo y lo medido en los ensayos llega al 15\%, los números estimados con el modelo dan ideas muy claras de lo que se puede esperar en cuanto a aceleración resistente perpendicular al plano de un muro de mampostería no reforzada. El autor considera que si se refinan un poco más algunos de los parámetros de entrada al modelo, como las resistencias máximas a cortante y a tracción de los ladrillos, producto de ensayos estándares sobre las propiedades mecánicas del mortero de pega y de los ladrillos, y algunas propiedades geométricas de difícil estimación como la constante torsional $\mathrm{K}_{\mathrm{T}}$, considerando por ejemplo la fisuración del mortero de pega, se logrará un mejor ajuste entre lo estimado y lo medido.

Es de destacar el buen ajuste que se puede lograr entre la aceleración resistente del muro medida del ensayo a escala bajo cargas inerciales del muro, y la aceleración predicha con base en el momento resistente que resulta del ensayo de la probeta. El ensayo propuesto hecho bajo las mismas condiciones del muro, que puede ser borde libre o soportado, puede homologarse para convertirlo en una medida directa de la aceleración resistente del muro.

En cuanto al modelo, más importante aún que el valor de la aceleración resistente predicha por éste, que es muy cercana a la medida, son las tendencias señaladas que permiten reconocer el tipo de participación que tienen las diferentes variables que intervienen en el fenómeno, que finalmente posibilita discernir entre las buenas y las malas prácticas constructivas y orientar algunas tendencias en la fabricación de los ladrillos de tal manera que se obtengan las mas altas resistencias con el mínimo de material. 


\section{APLICACIONES}

El modelo propuesto permite explicar algunos de los daños en edificaciones de mampostería no reforzada después de sismos intensos, al tiempo que llama la atención sobre algunas prácticas en la construcción de este sistema, que reducen considerablemente o anulan en algunos casos la resistencia de estos muros. Debe evitarse, entonces:

- La reducción del espesor de los muros, $t$. Esta dimensión, según se veía, está en proporción directa con la aceleración resistente.

- El incremento de la altura del ladrillo, w. Esta dimensión está en proporción inversa con la aceleración resistente.

- El incremento de las luces no soportadas, $L$. Esta dimensión al cuadrado está en proporción inversa a la aceleración resistente.

- Interrumpir la continuidad del mecanismo de transmisión de cargas en dirección horizontal por el empotramiento de líneas verticales de conducción (energía, agua, teléfono) o puertas y ventanas en los muros. Esta condición reduce a cero la aceleración resistente del muro.

- Transmitir cargas perpendiculares al plano del muro provenientes de otros elementos del sistema, como por ejemplo los techos. La resistencia de estos muros a cargas perpendiculares a su plano es apenas suficiente para transmitir sus propias cargas inerciales.

Igualmente, deben fomentarse las siguientes prácticas:

- Conexión de los bordes superiores de los muros. Las aceleraciones resistentes de los muros se disminuyen aproximadamente a la mitad por efecto del borde superior libre.

- Transmisión de cargas axiales a todos los muros de la edificación. El habilitar dos mecanismos de transmisión de cargas inerciales perpendiculares al plano del muro aumenta la capacidad resistente del muro.

- Cuidado especial del mortero de pega vertical que tradicionalmente se ha despreciado. Cerca del $60 \%$ de la aceleración resistente de los muros se debe al mecanismo de compresión del mortero de pega vertical.

En función de las dimensiones del ladrillo, de su densidad, de la resistencia máxima al cortante de los morteros de pega, de un factor de reducción de resistencia, $\phi_{R}$, y de la aceleración máxima para diseño en la zona, se puede definir la longitud máxima no soportada de los muros, si es que sólo se considera el mecanismo propuesto para transmisión de las cargas inerciales perpendiculares al plano del muro:

$$
L=\sqrt{\frac{8 \phi_{R} M_{R}}{\gamma \cdot t \cdot(w+b) \cdot\left(a_{R} / g\right)}}
$$

Por ejemplo, si se selecciona para cierta construcción un ladrillo y un mortero de pega como el utilizado en el segundo ensayo descrito, se tiene cuidado de eliminar los bordes libres en todos los muros, se acepta un factor de reducción de resistencia de 0.7 y se utiliza una aceleración 
para diseño de $0.6 \mathrm{~g}$, la longitud libre máxima no soportada de los muros sin carga axial sería de $5.2 \mathrm{~m}$.

Ahora, si a esta edificación se le agregan condiciones de borde libre en uno de los muros no cargueros y una pega vertical pobre o nula, la aceleración resistente, si se mantiene una longitud libre no soportada de $5.2 \mathrm{~m}$, se reduce a $0.11 \mathrm{~g}$.

\section{CONCLUSIONES}

El modelo propuesto para transmisión de cargas perpendiculares al plano en muros de mampostería no reforzada, verificado a través de ensayos a escala, impresiona por los valores tan altos de aceleraciones horizontales resistentes encontradas y al mismo tiempo llama la atención sobre prácticas comunes de construcción en muchos países, como por ejemplo el uso de muros muy esbeltos y de grandes claros en dirección horizontal, especialmente en Latinoamérica, que pueden reducir sustancialmente las resistencias encontradas a niveles peligrosos en términos de las aceleraciones probables en esas latitudes, e inclusive anular completamente las resistencias.

El contar con un modelo que explique las enormes diferencias en cuanto a daños reportados en edificaciones de mampostería no reforzada aparentemente iguales y después de sismos intensos, permite dirigir acertadamente, no sólo las reparaciones sino la construcción de nuevas edificaciones.

\section{AGRADECIMIENTOS}

El autor quiere expresar su más sincero agradecimiento a las dos instituciones que apoyaron el desarrollo de este trabajo, la Universidad EAFIT y la Universidad de Canterbury. En particular al Dr. José Ignacio Restrepo, director del laboratorio de estructuras de la segunda institución, sin cuyas ideas, comentarios y acompañamiento, este proyecto no sería una realidad. Al Dr. Félix Londoño, Director de investigación y docencia, y a la Lic. Diana Ospina, Jefe del Departamento de Compras de la primera institución, por su esfuerzo para hacer viable los ensayos de los muros con materiales colombianos en el laboratorio de la Universidad de Canterbury al otro lado del mundo. Igualmente, se quiere dejar constancia del desinteresado aporte de la ladrillera San Cristóbal de Medellín por la donación de los ladrillos para realizar los ensayos, y a la Federación Nacional de Cafeteros de Colombia por donar el transporte de los ladrillos desde Colombia hasta Nueva Zelanda.

Igualmente, el autor quieren agradecer el compromiso, sin el cual no hubiera sido posible la ejecución de los ensayos, de los técnicos del laboratorio de estructuras de la Universidad de Canterbury.

Finalmente, agradecer la participación en la dirección del último ensayo del estudiante de doctorado de la Universidad Politécnica de Cataluña, Ricardo Bonett, quien se desplazó desde España hasta Nueva Zelanda para colaborar en el desarrollo de este proyecto. 


\section{REFERENCIAS}

Abrams, D P, R Angel y J Uzarski (1993), “Transverse strength of damaged URM infills", The professional Journal of the Masonry Society, Vol. 12, No. 1, pp. 45-52.

Blaikie, T E L y R A Davey (2000), "Seismic behaviour of face loaded unreinforced masonry walls", Memorias, $12^{\text {th }}$ World Conference on Earthquake Engineering, Auckland, New Zealand, enero, Artículo No. 2574.

Doherty, T K, B Rodolico, N T K Lam, J L Wilson y M C Griffith (2000), "The modeling of earthquake induced collapse of unreinforced masonry walls combining force and displacement principals", Memorias, $12^{\text {th }}$ World Conference on Earthquake Engineering, Auckland, New Zealand, enero, Artículo No. 1645.

Gambarotta, L y L L Moneto (1999), "On the dynamic approach to the out of plane seismic collapse of masonry walls", Memorias, $8^{\text {th }}$ North American Masonry Conference, Austin, Texas, junio, Artículo No. 3D.2.

Kuzik, M D, J J R Cheng y A E Elwi (1999), "Full-scale out-of-plane cyclic tests of masonry walls with external GFRP sheets", Memorias, $8^{\text {th }}$ North American Masonry Conference, Austin, Texas, junio, Artículo No. 6A.2.

Lam, N T K, J L Wilson y G L Hutchinson (1995), "The seismic resistance of unreinforced masonry cantilever walls in low seismicity areas", Bulletin of the New Zealand National Society forEarthquake Engineering, Vol. 28, No. 3, pp. 179-195.

Mojsilovic, N y P Marti (1999), "Masonry subjected to combined actions: an overview", Memorias, $8^{\text {th }}$ North American Masonry Conference, Austin, Texas, junio, Artículo No. 1D.1.

Paulay, T y M J N Priestley (1992), Seismic design of reinforced concrete and masonry buildings, primera edición, John Wiley \& Sons, 744 pp.

Shultz, A E y J G Mueffelman (1999), "Design aspects for stability of masonry walls under outof-plane loading", Memorias, $8^{\text {th }}$ North American Masonry Conference, Austin, Texas, junio, Artículo No. 3D.1.

Timoshenko, S P y J N Goodier (1970), Theory of elasticity, tercera edición, McGraw-Hill, 567 pp. 